\title{
Evaluation of Deltoid Origin Status Following Open and Arthroscopic Repair of Large Rotator Cuff Tears: A Propensity- Matched Case-Control Study
}

\author{
Erica Kholinne ${ }^{1,2}$, Jae-Man Kwak², Yucheng Sun ${ }^{3}$, Hyojune Kim², Kyoung Hwan Koh ${ }^{2}$, In-Ho Jeon ${ }^{2}$ \\ ${ }^{1}$ Department of Orthopedic Surgery, St. Carolus Hospital, Jakarta, Indonesia \\ ${ }^{2}$ Department of Orthopedic Surgery, Asan Medical Center, University of Ulsan College of Medicine, Seoul, Korea \\ ${ }^{3}$ Department of Hand Surgery, Affiliated Hospital of Nantong University, Nantong University, Nantong, Jiangsu, China
}

\begin{abstract}
Background: The purpose of this study was to evaluate and compare deltoid origin status following large rotator cuff repair carried out using either an open or an arthroscopic method with a propensity score matching technique.

Methods: A retrospective review of 112 patients treated for full-thickness, large rotator cuff tear via either a classic open repair (open group) or an arthroscopic repair (arthroscopic group) was conducted. All patients included in the study had undergone postoperative magnetic resonance imaging (MRI) and clinical follow-up for at least 12 and 18 months after surgery, respectively. Propensity score matching was used to select controls matched for age, sex, body mass index, and affected site. There were 56 patients in each group, with a mean age of 63.3 years (range, 50-77 years). The postoperative functional and radiologic outcomes for both groups were compared. Radiologic evaluation for postoperative rotator cuff integrity and deltoid origin status was performed with 3-Tesla MRI.

Results: The deltoid origin thickness was significantly greater in the arthroscopic group when measured at the anterior acromion $(\mathrm{P}=0.006)$, anterior third $(\mathrm{P}=0.005)$, and middle third of the lateral border of the acromion level $(\mathrm{P}=0.005)$. The deltoid origin thickness at the posterior third of the lateral acromion was not significantly different between the arthroscopic and open groups. The arthroscopic group had significantly higher intact deltoid integrity with less scarring $(\mathrm{P}=0.04)$. There were no full-thickness deltoid tears in either the open or arthroscopic group.

Conclusions: Open rotator cuff repair resulted in a thinner deltoid origin, especially from the anterior acromion to the middle third of the lateral border of the acromion, at the 1-year postoperative MRI evaluation. Meticulous reattachment of the deltoid origin is as essential as rotator cuff repair when an open approach is selected.
\end{abstract}

Keywords: Propensity score; Rotator cuff; Arthroscopy; Magnetic resonance imaging

\section{INTRODUCTION}

Rotator cuff repair is one of the most commonly performed types of shoulder surgery. Improvements in arthroscopy techniques have shifted the preference for open repairs to all-arthroscopic rotator cuff procedures. All-arthroscopic rotator cuff repair has been accepted as the gold standard to treat rotator cuff repair [1,2]. An arthroscopic approach is favored by shoulder surgeons due to its

\section{Received: January 16, $2020 \quad$ Accepted: February 15, 2020}

Correspondence to: In-Ho Jeon

Department of Orthopedic Surgery, Asan Medical Center, University of Ulsan College of Medicine, 88 Olympic-ro 43-gil, Songpa-gu, Seoul 05505, Korea Tel: +82-2-3010-3896, Fax: +82-2-488-7877, E-mail: jeonchoi@gmail.com, ORCID: https://orcid.org/0000-0002-9289-9193

Financial support: This research was supported by the Convergence Technology Development Program for Bionic Arms through the National Research Foundation of Korea (NRF) funded by the Ministry of Science and ICT (No. 2014M3C1B2048422).

Conflict of interest: None. 
minimally invasive nature, which causes less insult to the deltoid muscle. Reducing trauma to the deltoid is believed to favor rehabilitation and therefore produce better clinical outcomes [3,4]. Despite movement toward all-arthroscopic rotator cuff repairs, open repairs are still being performed in cases where arthroscopic surgery is not feasible. In a traditional open repair of a large rotator cuff tear, deltoid detachment is performed to allow visualization of and access to the torn rotator cuff. The deltoid muscle is then repaired following rotator cuff repair. Previous studies have reported negative effects resulting from deltoid detachment that lead to more postoperative pain and poor shoulder function [5-7]. For this reason, the risk of deltoid insult is considered an indication for arthroscopic rotator cuff repair. Conversely, severe rotator cuff retraction and adhesion often complicate arthroscopic rotator cuff repair and may result in poor shoulder function [8-10].

Small rotator cuff tears are usually repairable using an arthroscopic technique, which typically produces satisfactory results. Medium rotator cuff repairs are suitable for both arthroscopic and open approaches, but there is less concern about deltoid injury due to the insignificant amount of deltoid detachment required in tears of this size. In contrast, massive rotator cuff tears are unpredictable regardless of the form of treatment, and they do not represent the most common type of pathology encountered [11,12]. Many studies have compared open and arthroscopic rotator cuff repair [1,11-17], but they have only compared the clinical outcomes and rotator cuff integrity with a non-matched arm, including all sizes of rotator cuff tears (small to massive) using a non-uniform surgical repair technique for open procedures $[12,14-16]$. We aimed to evaluate deltoid integrity following both open and arthroscopic repairs for large rotator cuff tear by comparing postoperative deltoid status. We hypothesized that (1) deltoid origin will change following large rotator cuff repair, and (2) open rotator cuff repairs cause more deltoid origin insults due to the re-attachment procedure required.

\section{METHODS}

This retrospective study was designed as a matched case control study that used a propensity score matching technique. It was approved by the Institutional Review Board of Asan Medical Center (IRB No. 2019-0787), and informed consent was waived due to retrospective nature of this study.

\section{Patient Selection}

We included 1,380 patients who underwent either an open or an arthroscopic rotator cuff repair between 2012 and 2016 in Asan Medical Center, Seoul, Korea. The inclusion criteria were (1) full thickness rotator cuff tear, (2) and primary repair, (3) in patients with at least one follow-up magnetic resonance imaging (MRI) scan performed 12 months after surgery, and (4) at least 18 months of clinical follow-up. The exclusion criteria were as follows: (1) incomplete medical data $(n=30),(2)$ previous surgery in the affected shoulder, (3) small $(<1 \mathrm{~cm})$, medium $(1-3 \mathrm{~cm})$, or massive tear $(>5 \mathrm{~cm}),(4)$ concurrent subscapularis tear, acromioclavicular arthritis that required concurrent distal clavicle resection, superior labral lesions that require concurrent repair, long head biceps pathology that required tenodesis, severe glenohumeral arthritis, anterior glenohumeral instability, (5) bilateral rotator cuff tears, or (6) worker's compensation case. Rotator cuff repair was performed by by (I.H.J) and (J.M.C).

\section{Surgical Technique}

Under general anesthesia, the patients were positioned in the beach-chair position and were given an interscalene block to reduce postoperative pain. Examinations under anesthesia were performed prior to the surgical procedure to assess passive range of motion (ROM).

\section{Arthroscopic Repair Technique}

A standard posterior portal was created $2 \mathrm{~cm}$ inferior to and $1 \mathrm{~cm}$ medial to the posterolateral acromion corner. The anterior portal through rotator interval was introduced using an outside-in technique. A standard diagnostic round was performed. The arthroscope was then introduced into the subacromial space to assess the acromion undersurface. An anterolateral acromioplasty was routinely performed in all patients. Afterward, a lateral portal was created under direct visualization with the help of a spinal needle; this portal later served as the main viewing portal. A bursectomy was carried out to expose the rotator cuff tear and shape. The mobility of the rotator cuff was evaluated with a retriever. The edge of the rotator cuff was refreshed and trimmed with an arthroscopic shaver and/or a punch. The size of the tear was measured mediolaterally. Greater tuberosity was then prepared with a burr for attaching the remnant tissue. The number of anchors used was dependent on the size of the rotator cuff tear and the repair configuration (single or double row). In the single-row repair configuration, the rotator cuff was routinely fixed using a bio-composite PEEK anchor (Helicoil PK 4.5 mm; Smith \& Nephew, Andover, MA, USA). In the double-row repair configuration, the rotator cuff was routinely fixed with a bio-composite PEEK (polyetheretherketone) anchor in a medial row (Helicoil PK $4.5 \mathrm{~mm}$, Smith \& Nephew) and lateral row (Footprint Ultra PK $4.5 \mathrm{~mm}$, Smith \& Nephew). An attempt was always made for tensionless repair with the maximum surface coverage of the footprint at the 
greater tuberosity.

\section{Open Repair Technique}

A 5-cm skin incision was made longitudinally starting from the mid-point of one-third of the lateral margin of the acromion to the lateral border of the coracoid process. The deltoid was split longitudinally about 3-4 cm between the anterior and middle deltoid. A curvilinear incision was made to take down a small portion of the anterior deltoid, and the coracoacromial ligament was peeled off from the undersurface of the acromial spur and preserved for later reattachment. An anterolateral acromioplasty was routinely performed with an oscillating saw. Multiple non-absorbable traction No. 2-0 Mersilk (Ethicon, Cincinnati, OH, USA) sutures were placed along the edge of the torn rotator cuff to assist in mobilization of the tendon. Gentle release of adhesion and removal of any bursal hypertrophy were carried out using Mayo scissors with respect of the remnant rotator cuffs. Once adequately mobilized, the margin was converged with multiple tendon-to-tendon sutures when necessary, and the torn edge of the tendon was reattached to the greater tuberosity by No. 2-0 Ethibond (Ethicon, Cincinnati, $\mathrm{OH}, \mathrm{USA}$ ) in a trans-osseous, double mattress fashion (Fig. 1). The deltoid was repaired along with the coracoacromial ligament to the acromion with heavy absorbable No. 1 Vicryl (Ethicon) suture.

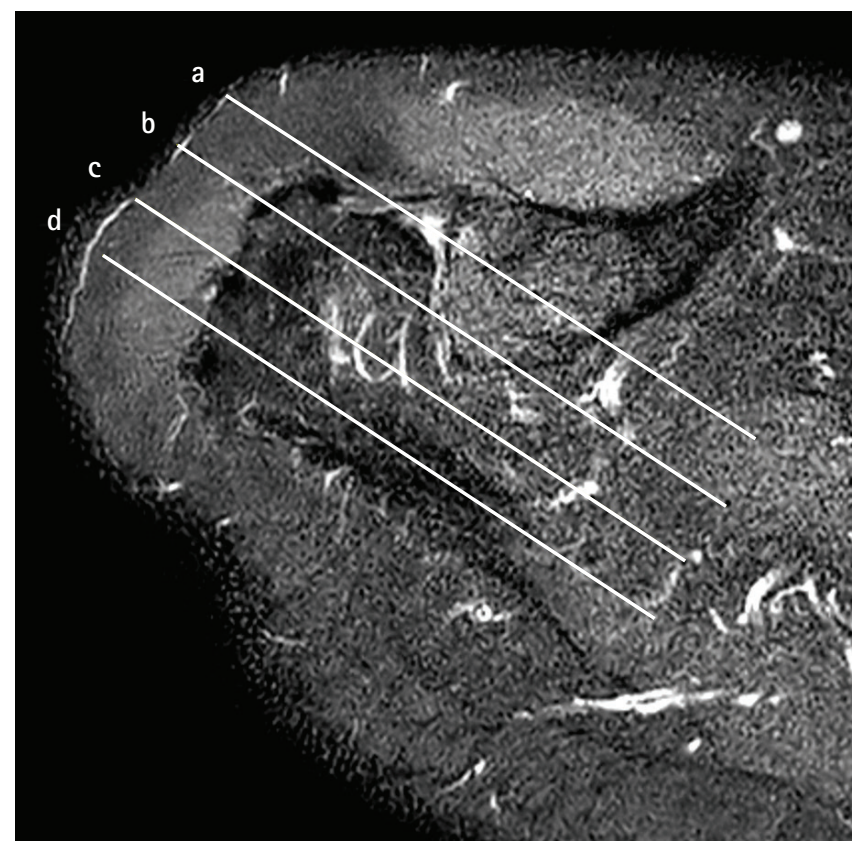

Fig. 1. Axial view of the shoulder magnetic resonance showed the region of interest for deltoid thickness measurement in four regions of interest: anterior acromion (a), anterior third (b), middle third (c), and posterior third (d) of the lateral acromion border according to scapular plane.

\section{Postoperative Protocol}

All patient arms remained in a sling for 6 weeks postoperatively, and only passive ROM was allowed during this time period. After 6 weeks, gradual full active motion was instituted, progressing to resistive strengthening, which was continued for three to 4 months. Heavy labor activities were restricted until at 6 months after surgery.

\section{Clinical Outcome Assessment}

An independent nurse practitioner documented the clinical assessment of pre-operative and postoperative parameters for (1) pain score with visual analog scale (VAS) score, (2) functional outcome with age-adjusted Constant score and American Shoulder and Elbow Surgeons (ASES) score, (3) ROM (forward elevation and external rotation) with a hand-held goniometer, and (4) muscle power (abduction and external rotator muscle strength) assessed with a myometer (Mecmesin Co., Nottingham, UK). Any complications that occurred following surgery were also recorded.

\section{Radiological Outcome Assessment}

All patients underwent radiological assessment with a 3-Tesla (3T) MRI at a minimum of one year following rotator cuff repair. The rotator cuff's integrity was evaluated using the method described by Sugaya et al. [18] The supraspinatus and infraspinatus were evaluated for any fatty infiltration according to the method of Fuchs et al. [19] The deltoid origin muscle thickness was assessed with MRI according to Gerber et al. [20] for integrity, scarring, and thickness. The deltoid origin thickness was measured in four zones: the anterior acromion and the anterior third, middle third, and posterior third at the inferior surface of the lateral acromion border according to the scapular plane (Fig. 1). Any discontinuity of any part of the deltoid origin on all sequences in sequential MRI slices was defined as a deltoid tear (Fig. 2). Scarring of the deltoid was confirmed as a high signal area on T1-weighted images with preserved integrity (Fig. 3). The postoperative thickness of the deltoid in each region of interest of the lateral acromion border was then compared to its preoperative measurement (Fig. 4). The evaluations were done independently by four shoulder-fellowship trained orthopedic surgeons (EK, JMK, HJK, DJP), and any discrepancies were resolved in a consensus meeting; if disagreement persisted, a senior shoulder surgeon who was not involved in the surgery (KHK) was consulted for the final assessment. All radiologic parameters were recorded both pre- and postoperatively.

\section{Statistical Analysis}

Sample size calculation with the a power of $90 \%$ and a 0.05 two-sid- 


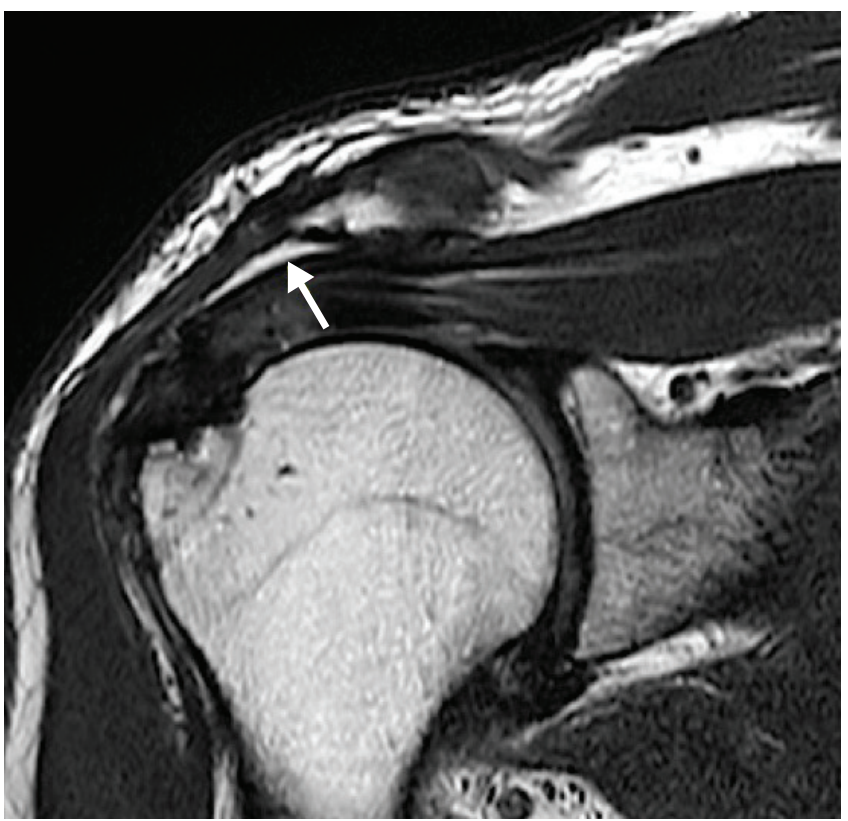

Fig. 2. Deltoid origin partial tear (arrow) 1 year after open rotator cuff repair.

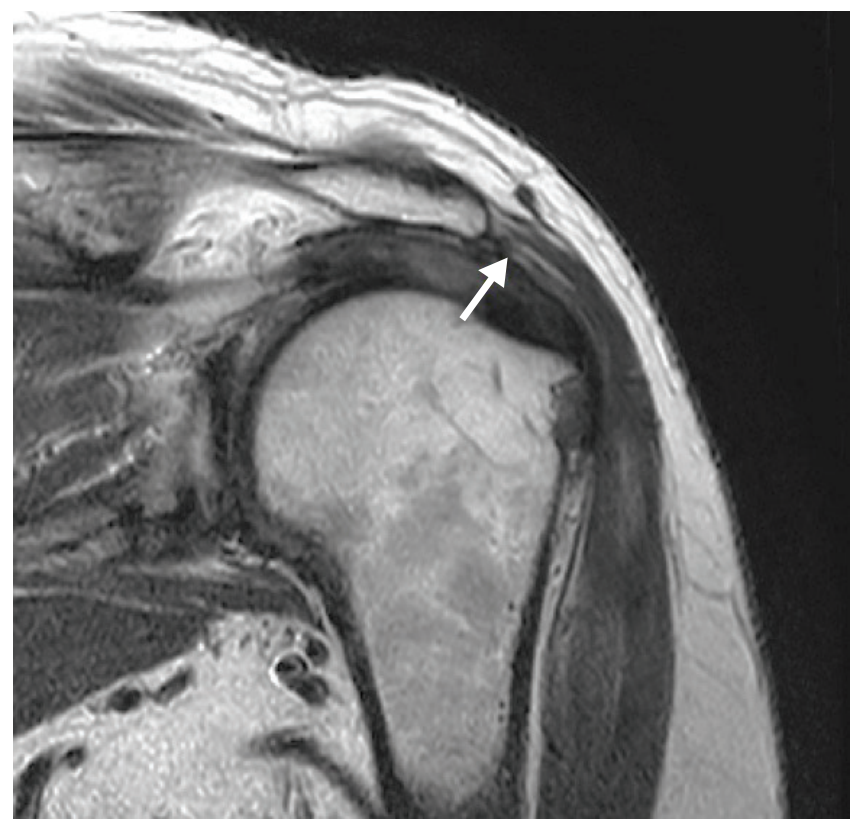

Fig. 3. Deltoid origin scarring following an open rotator cuff repair showing a high signal (arrow) at the interstitial layer of the deltoid origin.
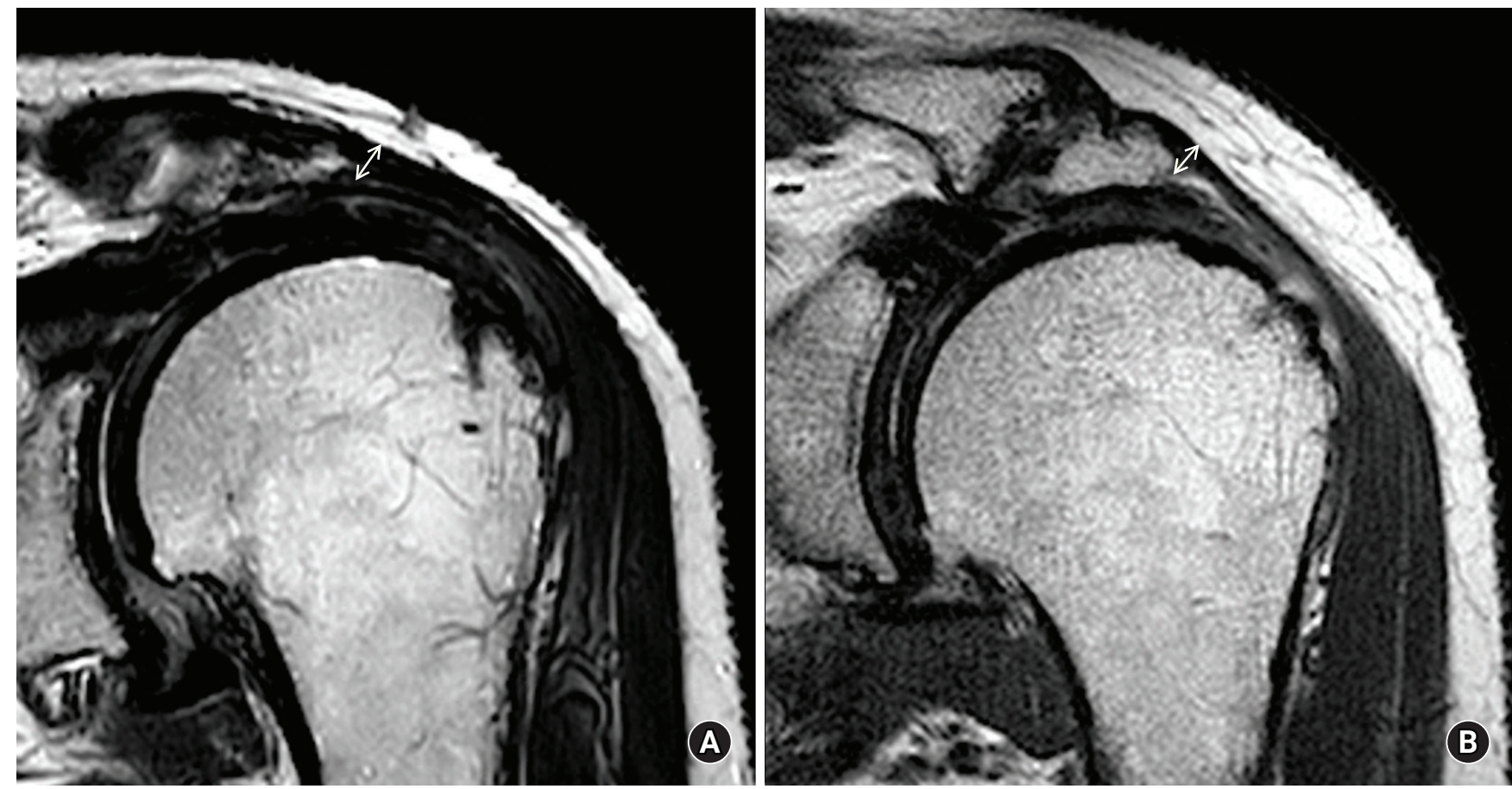

Fig. 4. (A) Measurement of the thickness of the deltoid origin (arrow) on magnetic resonance imaging (MRI) in the scapular plane prior to open rotator cuff surgery revealed the initial thickness. (B) An MRI evaluation 1 year after surgery showed a thin deltoid origin with a greater than $50 \%$ reduction in thickness (arrow) compared to the initial thickness.

ed significance level was performed with the minimum expected clinical importance difference in means of constant shoulder score for 10.4 points [21]. A minimum sample size of 47 patients in each group (including an extra 10\% due to the risk of loss to follow-up) was required. The propensity score matching technique was carried out with age, sex, affected shoulder, and body mass index as covariates. A total of 1,380 cases were recruited, but 30 were excluded because of incomplete medical data. Of the remaining $1,350 \mathrm{pa}-$ 
tients, 543 underwent open rotator cuff repair, while 837 underwent arthroscopic rotator cuff repair. Of the 543 open-repair patients, only 91 completed at least 18 months of clinical follow-up. Of these 91 patients, 56 had completed at least 1-year MRI and follow-up visits. The controls were the arthroscopic group that had been matched for age, sex, BMI, and affected side selected by propensity score matching (1:1 matching).

Tests for normality using the Kolmogorov-Smirnov method were applied to all datasets prior to statistical analysis. A Mann-Whitney U-test was used to compare any datasets with a skewed distribution, while an independent $\mathrm{t}$-test was carried out to compare the datasets with a normal distribution. The significance level was set at $\mathrm{P}<0.05$. Statistical analysis was performed using IBM SPSS ver. 23.0 (IBM Corp., Armonk, NY, USA) under the supervision of a biostatistician.

\section{RESULTS}

\section{Patient Demographics and Preoperative Baseline Data}

A total of 112 patients was included for analysis. The characteristics and preoperative baseline data of the open group and arthroscopic group are shown in Tables 1 and 2. There were no significant differences in demographic characteristics between these two matched groups.

\section{Clinical Outcome Assessment}

At the mean follow-up of 19.1 months, ROM, Constant score, ASES score, muscle power, and VAS were significantly improved following surgery. The arthroscopic group showed significantly better ROM $(\mathrm{P}=0.006)$ and VAS score $(\mathrm{P}<0.001)$ compared to the open group. In contrast, the open group demonstrated significantly better Constant and ASES scores $(\mathrm{P}=0.012$ and $\mathrm{P}=0.047$, respectively). The muscle powers for abduction and external rotation were superior in the open group, though there was no statistical difference ( $\mathrm{P}=0.068$ and $\mathrm{P}=0.182$, respectively). No complications were seen in either group. The postoperative clinical outcomes are shown in Table 3.

\section{Radiological Outcome Assessment}

Fatty infiltration of the supraspinatus and infraspinatus showed no significant difference between the two groups. The retear rate was higher in the arthroscopic group (21.4\%) compared to the open group (17.8\%), though the difference was not statistically significant $(\mathrm{P}=0.300)$. No patient required revision surgery (even in the case of a cuff tendon re-tear and deltoid injury) at the final follow-up as all were in an asymptomatic state. The deltoid origin thickness was significantly greater in the arthroscopic group when measured at the anterior acromion area $(6.2 \pm 1.4$ vs. $4.9 \pm 1.1 \mathrm{~mm}$, $\mathrm{P}=0.006)$, anterior third ( $6.3 \pm 1.3$ vs. $4.5 \pm 0.9 \mathrm{~mm}, \mathrm{P}=0.005)$, and middle third of the lateral acromion border $(6.7 \pm 1.3$ vs. $4.6 \pm 1.0$ $\mathrm{mm}, \mathrm{P}=0.005)$. The arthroscopic group had significantly higher intact deltoid integrity with less scarring $(\mathrm{P}=0.04)$. There were no full-thickness deltoid tears in our observations for both open and arthroscopic groups. The postoperative thickness of the deltoid insertion was significantly maintained with less than 50\% reduction from its preoperative thickness in the arthroscopy group $(80.7 \%)$

Table 1. Baseline demographics for both groups

\begin{tabular}{|c|c|c|c|}
\hline Variable & Open group $(n=56)$ & Arthroscopic group $(n=56)$ & P-value \\
\hline Age (yr) & $63.66 \pm 7.97$ & $61.56 \pm 5.51$ & 0.111 \\
\hline Sex & & & 0.109 \\
\hline Female & $37(66.7)$ & $33(59.3)$ & \\
\hline Male & $19(33.3)$ & $23(40.7)$ & \\
\hline Affected shoulder & & & 0.06 \\
\hline Right & $43(77.8)$ & $48(85.2)$ & \\
\hline Left & $13(22.2)$ & $8(14.8)$ & \\
\hline Body mass index $\left(\mathrm{kg} / \mathrm{m}^{2}\right)$ & & & 0.805 \\
\hline Underweight $(<18.5)$ & 0 & 0 & \\
\hline Normal (18.5-24.9) & $29(51)$ & $25(44.6)$ & \\
\hline Overweight (25.0-29.9) & $27(49)$ & $28(50)$ & \\
\hline Obese class I (30.0-34.9) & 0 & $3(5.4)$ & \\
\hline Obese class II (35.0-39.9) & 0 & 0 & \\
\hline Obese class III (40.0) & 0 & 0 & \\
\hline Comorbidity & $24(42.9)$ & $29(52.7)$ & 0.302 \\
\hline
\end{tabular}

Values are presented as mean \pm standard error or number (\%). 
Table 2. Preoperative clinical and radiologic data for both groups

\begin{tabular}{|c|c|c|c|}
\hline Variable & Open group & Arthroscopic group & P-value ${ }^{a)}$ \\
\hline Presence of shoulder stiffness & $12(21.4)$ & $12(21.4)$ & 1.000 \\
\hline Presence of shoulder trauma & $16(28.5)$ & $15(26.7)$ & 0.569 \\
\hline \multicolumn{4}{|l|}{ ROM } \\
\hline $\mathrm{FE}$ & $139.63 \pm 5.18$ & $144.26 \pm 3.63$ & 0.468 \\
\hline ER & $43.52 \pm 4.12$ & $43.15 \pm 7.74$ & 0.827 \\
\hline \multicolumn{4}{|l|}{ Functional score } \\
\hline Constant score & $54.11 \pm 3.63$ & $56.15 \pm 2.75$ & 0.657 \\
\hline ASES score & $57.15 \pm 3.91$ & $58.63 \pm 3.32$ & 0.774 \\
\hline \multicolumn{4}{|l|}{ Muscle power } \\
\hline Abduction & $3.63 \pm 0.37$ & $2.85 \pm 0.35$ & 0.156 \\
\hline ER & $4.05 \pm 0.37$ & $3.16 \pm 0.27$ & 0.58 \\
\hline Pain VAS & $4.89 \pm 0.35$ & $5.59 \pm 0.31$ & 0.14 \\
\hline \multicolumn{4}{|l|}{ Fatty infiltration } \\
\hline Supraspinatus & $0.4 \pm 0.65$ & $0.78 \pm 0.71$ & 0.192 \\
\hline Infraspinatus & $0.5 \pm 0.7$ & $0.77 \pm 0.5$ & 0.283 \\
\hline \multicolumn{4}{|l|}{ Deltoid muscle thickness (mm) } \\
\hline At anterior acromion area & $6.9 \pm 0.9$ & $7.0 \pm 0.7$ & 0.246 \\
\hline At anterior third of lateral acromion border & $6.6 \pm 0.8$ & $6.6 \pm 0.7$ & 0.171 \\
\hline At middle third of lateral acromion border & $6.6 \pm 0.9$ & $6.5 \pm 0.8$ & 0.264 \\
\hline At posterior third of lateral acromion border & $6.8 \pm 1.1$ & $7.0 \pm 0.8$ & 0.289 \\
\hline
\end{tabular}

Values are presented as number (\%) or mean \pm standard error. ROM, range of motion; FE, forward elevation; ER, external rotation; ASES, American Shoulder and Elbow Surgeons score; VAS, visual analog scale. ${ }^{\text {a) }}$ Significant level, $\mathrm{P}<0.05$.

compared to the open group $(64.1 \% ; \mathrm{P}=0.04)$.

\section{DISCUSSION}

The current study showed that there was a difference in postoperative deltoid status between arthroscopic and open repairs of large rotator cuff tears. The deltoid origin thickness was reduced to more than $50 \%$ of its preoperative thickness in 19 of 56 patients (33.9\%) who underwent open rotator cuff repair. In contrast, deltoid origin thickness was preserved within $50 \%$ of its preoperative thickness in 43 of 56 patients (76.7\%) who underwent arthroscopic rotator cuff repair. The clinical outcomes for abduction muscle power were not influenced by deltoid function. The current study found that open rotator cuff repair resulted in better functional outcomes of Constant and ASES scores as measured variables. The minimal clinically important differences were a Constant score of 10.4 [22] and a range from 12 to 17 for ASES score [23]. Despite significant superiority in functional outcomes following open rotator cuff repair, the differences between groups did not exceed the smallest amount to be meaningful; therefore, this finding is disregarded and considered as comparable outcomes [24]. Open rotator cuff repair procedures for large rotator cuff tears caused more del- toid origin injury and had no significant influence on clinical outcomes due to the meticulous re-attachment procedure required. Therefore, reattachment of the deltoid origin is essential when performing open rotator cuff repair surgery.

The current study found that the incidence of deltoid origin tear following rotator cuff repair was 5.4\%. However, this rate was inconsistent with previous studies because varying sizes of rotator cuff tears were included, from large to massive $[25,26]$. Our study focused on large-sized rotator cuff tears, so we avoided different amounts of deltoid detachment during open surgery. This approach enabled us to confirm a definitive incidence for deltoid origin tear.

The integrity of rotator cuff repair and deltoid origin reattachment procedures performed at our center were evaluated using MRI due to the unpredictability of ultrasonographic examinations [27]. The MRI evaluation was carried out 1 year following surgery with a 3T scanner in our study. We believe that both timing and MRI magnitude may play a role in evaluating deltoid origin status. A previous study obtained MRI evaluations with a 1.5-Tesla scanner 6 months after surgery [25]. We think that a longer interval between repair and MRI provides a better evaluation of the disease course, and a higher MRI magnitude increases the sensitivity, 
Table 3. Postoperative clinical and radiologic data for both groups

\begin{tabular}{|c|c|c|c|}
\hline Variable & Open group & Arthroscopic group & P-value \\
\hline \multicolumn{4}{|l|}{ ROM } \\
\hline FE & $151.85 \pm 0.93$ & $158.33 \pm 2.05$ & $0.006^{\mathrm{a})}$ \\
\hline ER & $44.26 \pm 0.34$ & $50.93 \pm 2.18$ & $0.002^{\mathrm{a})}$ \\
\hline \multicolumn{4}{|l|}{ Functional score } \\
\hline Constant score & $75.93 \pm 1.88$ & $69.59 \pm 1.53$ & $0.012^{\mathrm{a})}$ \\
\hline ASES score & $89.19 \pm 1.36$ & $85.33 \pm 1.32$ & $0.047^{\mathrm{a})}$ \\
\hline \multicolumn{4}{|l|}{ Muscle power $(\mathrm{kg})$} \\
\hline Abduction & $4.47 \pm 0.43$ & $3.49 \pm 1.51$ & 0.068 \\
\hline ER & $4.47 \pm 0.36$ & $3.92 \pm 0.18$ & 0.182 \\
\hline Pain VAS & $1.78 \pm 0.21$ & $0.59 \pm 0.15$ & $<0.001^{\mathrm{a})}$ \\
\hline Fatty infiltration & $0.35 \pm 0.58$ & $0.51 \pm 0.60$ & 0.181 \\
\hline \multicolumn{4}{|l|}{ Supraspinatus } \\
\hline Infraspinatus & $0.55 \pm 0.70$ & $0.67 \pm 0.50$ & 0.182 \\
\hline Postoperative rotator cuff integrity & & & 0.642 \\
\hline Sugaya type I & $28(50)$ & $26(47.3)$ & \\
\hline Sugaya type II & $18(32.1)$ & $17(30.9)$ & \\
\hline Sugaya type III & $8(14.3)$ & $8(14.5)$ & \\
\hline Sugaya type IV & 0 & $4(7.3)$ & \\
\hline Sugaya type V & $2(3.6)$ & 0 & \\
\hline \multicolumn{4}{|l|}{ Deltoid origin thickness (mm) } \\
\hline At anterior acromion area & $4.9 \pm 1.1$ & $6.2 \pm 1.4$ & $0.006^{\mathrm{a})}$ \\
\hline At anterior third of lateral acromion border & $4.5 \pm 0.9$ & $6.3 \pm 1.3$ & $0.005^{\mathrm{a})}$ \\
\hline At middle third of lateral acromion border & $4.6 \pm 1.0$ & $6.7 \pm 1.3$ & $0.005^{\mathrm{a})}$ \\
\hline At posterior third of lateral acromion border & $6.1 \pm 1.2$ & $6.8 \pm 1.4$ & 0.354 \\
\hline \multicolumn{4}{|l|}{ Postoperative deltoid MRI status } \\
\hline Intact & $38(67.9)$ & $40(72.7)$ & $0.04^{\mathrm{a})}$ \\
\hline Scarring & $15(26.8)$ & $12(21.8)$ & \\
\hline Partial thickness tear & $3(5.4)$ & $3(5.4)$ & \\
\hline Postoperative deltoid insertion thickness & & & $0.04^{\text {a) }}$ \\
\hline$<50 \%$ reduce & $34(66.1)$ & $43(76.7)$ & \\
\hline$>50 \%$ reduce & $19(33.9)$ & $10(23.3)$ & \\
\hline
\end{tabular}

Values are presented as mean \pm standard error or number (\%).

ROM, range of motion; FE, forward elevation; ER, external rotation; ASES, American Shoulder and Elbow Surgeons score; VAS, visual analog scale. ${ }^{\text {a) }}$ Significant level, $\mathrm{P}<0.05$.

which allows to detection of structural changes of deltoid origin and thus avoids underestimation of deltoid origin injury compared to an MRI scanner with a lower magnitude. This approach may explain why our findings produced a larger number of deltoid origin tears.

The deltoid origin thickness was measured regionally and was greater in the anterior acromion area and the anterior and middle thirds of the lateral acromion border area following arthroscopic rotator cuff repair. However, the deltoid thickness at the posterior third of the lateral acromion border was similar in the open and the arthroscopic groups. Standard anterolateral acromioplasty was used in all patients who underwent arthroscopic rotator cuff re- pair. In this technique, the amount of resection was limited to the anterior third of the lateral acromion border [28]. Therefore, the likelihood of injuring the deltoid origin will depend upon the extent of acromioplasty required. Anterolateral acromioplasty was also applied in all open rotator cuff repairs in our study. Nevertheless, we think that the extent of deltoid detachment needed to achieve cuff visualization was not consistent due to the variation in tear location and retraction level. A greater amount of deltoid origin involvement had a substantial influence upon the re-attachment procedure in open rotator cuff repair.

The current study had several strengths. First, we only included large rotator cuff tears and conducted an appropriate power analy- 
sis prior to the study. Second, a 3T MRI was used to evaluate both pre- and postoperative deltoid origin status. Third, this study ensured the inclusion of a matching case and control group by using propensity score matching to balance the clinical characteristics of the groups and therefore allow more accurate comparisons within observational studies by simulating a randomized controlled trial [29]. The matching technique, which included patient age, sex, affected site, and BMI, ensured assignment of a control patient to each case. This is a major advantage of the frequency matching technique in which the nearest neighbors are selected for each case to serve as a control despite any slight differences in the matching variable distribution. Because of potential residual confounding, regression models were also controlled for age, sex, and BMI [1530]. Fourth, this study showed that attention should be given to the deltoid origin at the anterior acromion to the middle third of the lateral acromion border in open rotator cuff repairs since the deltoid origin is thinner in this area. Therefore, we advocate that surgeons utilize an adequate amount of the deltoid origin tendon during the reattachment procedure.

\section{Limitations}

The most significant limitation of this study is its retrospective nature. The included population was ideal for a clinical study, but it still may limit extrapolation of our findings to a general population, leading to selective bias in this study. This study also only included large tears, which limited translation of our results to all rotator cuff tear sizes. Despite these limitations, we attempted to minimize bias by excluding small, medium, and massive rotator cuff tears from our study design to provide a straightforward result. This study compared the clinical outcomes at the final follow-up visit as opposed to the postoperative timeline. MRI was used to evaluate deltoid origin status; however, the surgical repair itself may result in muscle scarring and thinning, which can lead to false positives on MRI scans. Nevertheless, despite these limitations, the accuracy provided by a 3T MRI scanner should minimize the incidence of false positives. One additional limitation was that it is difficult to blind the radiologic evaluation process due to the holes drilled in the acromion following open rotator cuff repair. Lastly, we suggest that future studies develop a better methodology to evaluate the deltoid status postoperatively.

The current study showed that there was a change in deltoid origin status following both open and arthroscopic repairs of large rotator cuff tears. Open rotator cuff repair resulted in a thinner deltoid origin, especially in the anterior acromion to the middle third of the lateral border of the acromion at the 1-year postoperative MRI evaluation. Meticulous reattachment of the deltoid origin is as essential as a proper rotator cuff repair for the open procedure.

\section{ACKNOWLEDGMENTS}

We thank Jessica Kholinne for providing medical illustrations and Jong Hwee Park and Dongjun Park for their assistance in collecting medical data.

\section{ORCID}

Erica Kholinne https://orcid.org/0000-0002-4326-8205 Jae-Man Kwak https://orcid.org/0000-0002-4395-8345 Yucheng Sun https://orcid.org/0000-0001-5899-2792

Hyojune Kim Kyoung Hwan Koh In-Ho Jeon https://orcid.org/0000-0001-7665-536X https://orcid.org/0000-0002-6181-9621 https://orcid.org/0000-0002-9289-9193

\section{REFERENCES}

1. Bayle X, Pham TT, Faruch M, Gobet A, Mansat P, Bonnevialle N. No difference in outcome for open versus arthroscopic rotator cuff repair: a prospective comparative trial. Arch Orthop Trauma Surg 2017;137:1707-12.

2. Acevedo DC, Paxton ES, Williams GR, Abboud JA. A survey of expert opinion regarding rotator cuff repair. J Bone Joint Surg Am 2014;96:e123.

3. Snyder SJ. Evaluation and treatment of the rotator cuff. Orthop Clin North Am 1993;24:173-92.

4. Gartsman GM, Khan M, Hammerman SM. Arthroscopic repair of full-thickness tears of the rotator cuff. J Bone Joint Surg Am 1998;80:832-40.

5. Baker CL, Liu SH. Comparison of open and arthroscopically assisted rotator cuff repairs. Am J Sports Med 1995;23:99-104.

6. Fuchs B, Gilbart MK, Hodler J, Gerber C. Clinical and structural results of open repair of an isolated one-tendon tear of the rotator cuff. J Bone Joint Surg Am 2006;88:309-16.

7. Mormino MA, Gross RM, McCarthy JA. Captured shoulder: a complication of rotator cuff surgery. Arthroscopy 1996;12:45761.

8. Berth A, Neumann W, Awiszus F, Pap G. Massive rotator cuff tears: functional outcome after debridement or arthroscopic partial repair. J Orthop Traumatol 2010;11:13-20.

9. Verma NN, Dunn W, Adler RS, et al. All-arthroscopic versus mini-open rotator cuff repair: a retrospective review with minimum 2-year follow-up. Arthroscopy 2006;22:587-94.

10. Yoo JC, Ahn JH, Koh KH, Lim KS. Rotator cuff integrity after arthroscopic repair for large tears with less-than-optimal footprint coverage. Arthroscopy 2009;25:1093-100.

11. Kim SH, Ha KI, Park JH, Kang JS, Oh SK, Oh I. Arthroscopic 
versus mini-open salvage repair of the rotator cuff tear: outcome analysis at 2 to 6 years' follow-up. Arthroscopy 2003;19:746-54.

12. Ide J, Maeda S, Takagi K. A comparison of arthroscopic and open rotator cuff repair. Arthroscopy 2005;21:1090-8.

13. Bishop J, Klepps S, Lo IK, Bird J, Gladstone JN, Flatow EL. Cuff integrity after arthroscopic versus open rotator cuff repair: a prospective study. J Shoulder Elbow Surg 2006;15:290-9.

14. Buess E, Steuber KU, Waibl B. Open versus arthroscopic rotator cuff repair: a comparative view of 96 cases. Arthroscopy 2005;21:597-604.

15. Gwark JY, Sung CM, Na JB, Park HB. Outcomes of arthroscopic rotator cuff repair in patients who are 70 years of age or older versus under 70 years of age: a sex- and tear size-matched case-control study. Arthroscopy 2018;34:2045-53.

16. Severud EL, Ruotolo C, Abbott DD, Nottage WM. All-arthroscopic versus mini-open rotator cuff repair: A long-term retrospective outcome comparison. Arthroscopy 2003;19:234-8.

17. Walton JR, Murrell GA. A two-year clinical outcomes study of 400 patients, comparing open surgery and arthroscopy for rotator cuff repair. Bone Joint Res 2012;1:210-7.

18. Sugaya H, Maeda K, Matsuki K, Moriishi J. Repair integrity and functional outcome after arthroscopic double-row rotator cuff repair: a prospective outcome study. J Bone Joint Surg Am 2007; 89:953-60.

19. Fuchs B, Weishaupt D, Zanetti M, Hodler J, Gerber C. Fatty degeneration of the muscles of the rotator cuff: assessment by computed tomography versus magnetic resonance imaging. J Shoulder Elbow Surg 1999;8:599-605.

20. Gerber C, Catanzaro S, Betz M, Ernstbrunner L. Arthroscopic correction of the critical shoulder angle through lateral acromioplasty: a safe adjunct to rotator cuff repair. Arthroscopy 2018; 34:771-80.

21. Wylie JD, Beckmann JT, Granger E, Tashjian RZ. Functional outcomes assessment in shoulder surgery. World J Orthop 2014;
5:623-33.

22. Kukkonen J, Kauko T, Vahlberg T, Joukainen A, Aarimaa V. Investigating minimal clinically important difference for Constant score in patients undergoing rotator cuff surgery. J Shoulder Elbow Surg 2013;22:1650-5.

23. Tashjian RZ, Deloach J, Porucznik CA, Powell AP. Minimal clinically important differences (MCID) and patient acceptable symptomatic state (PASS) for visual analog scales (VAS) measuring pain in patients treated for rotator cuff disease. J Shoulder Elbow Surg 2009;18:927-32.

24. McGlothlin AE, Lewis RJ. Minimal clinically important difference: defining what really matters to patients. JAMA 2014; 312:1342-3.

25. Cho NS, Cha SW, Rhee YG. Alterations of the deltoid muscle after open versus arthroscopic rotator cuff repair. Am J Sports Med 2015;43:2927-34.

26. Gumina S, Di Giorgio G, Perugia D, Postacchini F. Deltoid detachment consequent to open surgical repair of massive rotator cuff tears. Int Orthop 2008;32:81-4.

27. de Jesus JO, Parker L, Frangos AJ, Nazarian LN. Accuracy of MRI, MR arthrography, and ultrasound in the diagnosis of rotator cuff tears: a meta-analysis. AJR Am J Roentgenol 2009;192:1701-7.

28. Katthagen JC, Marchetti DC, Tahal DS, Turnbull TL, Millett PJ. The effects of arthroscopic lateral acromioplasty on the critical shoulder angle and the anterolateral deltoid origin: an anatomic cadaveric study. Arthroscopy 2016;32:569-75.

29. McDonald RJ, McDonald JS, Kallmes DF, Carter RE. Behind the numbers: propensity score analysis-a primer for the diagnostic radiologist. Radiology 2013;269:640-5.

30. Kessler KE, Robbins CB, Bedi A, Carpenter JE, Gagnier JJ, Miller BS. Does increased body mass index influence outcomes after rotator cuff repair. Arthroscopy 2018;34:754-61. 